\title{
Health disparity toward noncommunicable diseases among residents in rural Cambodia: a descriptive study
}

\author{
Yurie Kobashi ${ }^{1,2}$, Hong Chhay ${ }^{1}$, Thyryfong Savat ${ }^{3}$, Manabu Okawada ${ }^{4}$, \\ Masaharu Tsubokura ${ }^{2}$, and Yushifumi Hayashi ${ }^{3}$ \\ ${ }^{1}$ Department of General Internal Medicine, Sunrise Japan Hospital Phnom Penh, Cambodia \\ ${ }^{2}$ Department of Public Health, Fukushima Medical University School of Medicine, Japan \\ ${ }^{3}$ Department of Neurosurgery, Sunrise Japan Hospital Phnom Penh, Cambodia \\ ${ }^{4}$ Department of Pediatrics, Sunrise Japan Hospital Phnom Penh, Cambodia
}

\begin{abstract}
Objective: Regional disparities in health services is a crucial problem in Cambodia. Particularly, a number of NCD risk factors are more common among the rural poor. Fortunately, $80 \%$ of NCDs are preventable and cost-effective interventions exist. Therefore, health care needs assessments regarding NCDs in poor rural areas are vital. The object of this pilot study was to identify health care needs regarding NCDs among residents in poor rural areas in Cambodia.

Materials and Methods: A medical health check-up and questionnaire survey were conducted with 208 rural residents who participated in a free health check-up and doctor consultation in Kaoh Peam Reang.

Results: One hundred sixteen (55.8\%) females were included in the total sample size of 208 participants; the majority (52.9\%) were between 35 and 65 years of age. Twenty-five participants (12\%) were current smokers, and 44 (21\%) were current alcohol drinkers. Eighty (38.5\%) participants had hypertension and 44 participants (21.2\%) had a body mass index over 25. Alcohol drinking and smoking habits were more common among men. The five most frequent medical complaints were headache (18.3\%), lower back pain (14.4\%), foot and hand pain (13.9\%), joint pain (10.1\%), and difficulty breathing (10.1\%).

Conclusions: The medical need for doctor consultations regarding chronic disease and chronic pain might be higher in poor rural areas in Cambodia.
\end{abstract}

Key words: regional disparity, global health, noncommunicable disease

\section{Introduction}

Reducing regional disparities is a significant public health issue to ensure that essential medical care is provided to all people ${ }^{1}$. In particular, proper evaluation of medical needs is essential in reducing regional disparities ${ }^{1)}$. Noncommunicable diseases (NCDs) are the most common cause of death worldwide ${ }^{2-4)}$, and more than $90 \%$ of premature deaths from

Received: May 21, 2020

Accepted: June 25, 2020

Correspondence: Yurie Kobashi, Department of Public Health, Fukushima Medical University School of Medicine, Fukushima City, Fukushima 960-1295, Japan E-mail: yurie-s@umin.ac.jp

This is an open-access article distributed under the terms of the Creative Commons Attribution Non-Commercial No Derivatives (by-nc-nd) License $<\mathrm{http}: / /$ creativecommons.org/ licenses/by-nc-nd/4.0/>.
NCDs occur in low- and middle-income countries (LMIC) ${ }^{2}$.

Cambodia is classified as an LMIC by the World Bank, and regional disparities in health services is a crucial problem in Cambodia ${ }^{5,6)}$. Eighty-four percent of the nation's wealthy population live in Phnom Penh, the capital city ${ }^{5}$. Indicators of health needs, such as the mortality rate of children under five years of age, are approximately three times higher in rural areas than in urban areas ${ }^{5}$. Further, the Cambodian healthcare system is already flooded with more NCD patients than it is able to treat ${ }^{7)}$. A number of NCD risk factors are more common among the poor rural population compared to urban locations, including tobacco use, solid fuel use, insufficient fruit and vegetable consumption, and harmful use of alcohol ${ }^{8)}$. Fortunately, $80 \%$ of NCDs are preventable and a range of highly cost-effective interventions exist ${ }^{8)}$. Therefore, needs assessments with respect to NCDs in poor rural areas are vital.

This study aimed to identify health care needs regarding NCDs among residents in poor rural areas in Cambo- 
dia, with a view to developing strategies to reduce regional health disparities.

\section{Method}

This study used a cross-sectional descriptive survey design.

\section{Location of the study}

Kaoh Peam Reang is an island located in the Mekong River in the Prey Veng province of Cambodia. It covers an area of $7.14 \mathrm{~km}^{2}$ and has a population of approximately 1,155 . The area is located 86 kilometers southeast of Phnom Penh, and the climate, according to the Köppen climate classification, is tropical monsoon. The residents live using boats, which increases their difficulties in accessing essential medical care. Almost all the residents are engaged in agriculture and fishery. Since most people are living under the poverty line, the island is often a target for social service or volunteer work. There is one health center on the island; however, it is mostly non-functional. There are no other health facilities.

\section{Free health check-up}

This free health check-up is managed by Sunrise Japan Hospital (SJH), a private hospital in Phnom Penh that has 14 medical departments, 50 beds for inpatients, an intensive care unit, and endovascular treatment facilities. Most patients who visit the hospital are from the area surrounding Phnom Penh. SJH, which opened in 2016, aims to provide Japanese-standard quality medical care in Cambodia. The hospital has 213 employees, including Cambodian and Japanese staff as well as staff from other countries. SJH has treated 100,000 patients since it opened.

\section{Participant recruitment}

SJH arranged to conduct free health check-ups to improve rural residents' health and to promote the hospital, as part of its social responsibility activity. To decide on the location and to recruit participants, we solicited the help of a collaborator who is engaged in social responsibility activities in Kaoh Peam Reang. The collaborator requested an influential local person in Kaoh Peam Reang to recruit participants. This local person announced the details (date, time, place, contents) of the free health check-ups for residents. The check-up included education about diabetes, measurement of weight, height, and body temperature, and consultation with a doctor for those individuals who had a health problem. The check-ups were conducted at a meetinghouse in the center of the island. The medical consultations were conducted by approximately 10 doctors from SJH. The doctors were Cambodian and Japanese and included both genders. Two hundred eight residents participated in this study.

\section{Survey tool}

The questionnaire developed for the participants in the free medical health check-up was based on a general questionnaire that is used at $\mathrm{SJH}$ for a general feasibility health survey in rural areas. Medical doctors were consulted about the contents of the questionnaire. The instrument included questions about age, gender, past medical history, medical drug use, alcohol and tobacco habits, and respondents' chief medical complaint. Physicians made diagnoses based on a medical interview and physical examination, and prescribed medication as needed.

\section{Procedure}

This study was undertaken in December 2019. The questionnaire included an explanation written in Khmer language that the data would be used for research and improvement of residents' health. First, a nurse from SJH collected the questionnaire and conducted a brief interview with each patient to assess personal data. Participants then underwent a consultation with a doctor, which included a physical examination. If the doctor made a diagnosis, he or she provided advice to participants.

\section{Data analysis}

The characteristics of participants who attended a doctor consultation were analyzed descriptively. Hypertension was defined as systolic blood pressure above 140 or diastolic blood pressure above 90. Stratified analyses of age and gender were conducted. In addition, patients' major complaints and diagnoses made by the doctors were analyzed descriptively. Analysis was performed using STATA/IC (Lightstone, Texas USA, version15) and Excel. This study was approved by the National Ethics Committee for Health Research (approval number 078NECHR).

\section{Results}

Table 1 presents the characteristics of the participants in this study. A total of 208 individuals, including 116 females $(55.8 \%)$, participated in this research. Sixty-one participants $(29.3 \%)$ had a past medical history, and 35 participants (16.8\%) were taking prescribed or over-the-counter medication daily. A total of 25 participants (12.0\%) smoked tobacco, and 44 participants (21.2\%) consumed alcohol daily. Eighty participants (38.5\%) had hypertension.

Table 2 shows the rate of alcohol and smoking habits, hypertension, and BMI (body mass index) in each age and gender group. Two-thirds of people (66.7\%) in the 35-49 age group were current alcohol drinkers and $24.2 \%$ percent of people in this age group had a smoking habit. Alcohol and tobacco consumption were higher among males than females $(P<0.001$; Fisher's exact test). The rate of hypertension was also higher among males than females $(P<0.001$; 
Table 1 Characteristics of participants living in Kaoh Peam Reang area, Prey Veng province, Cambodia in $2019(n=208)$

\begin{tabular}{|c|c|}
\hline & n (\%) \\
\hline \multicolumn{2}{|l|}{ Gender } \\
\hline Male & $85(40.9)$ \\
\hline Female & $116(55.8)$ \\
\hline Unknown & $7(3.4)$ \\
\hline \multicolumn{2}{|l|}{ Age } \\
\hline$<19$ & $15(7.2)$ \\
\hline $20-34$ & $24(11.5)$ \\
\hline $35-49$ & $45(21.6)$ \\
\hline $50-64$ & $65(31.3)$ \\
\hline $65-80$ & $49(23.6)$ \\
\hline $80<$ & 4 (1.9) \\
\hline Unknown & $6(2.9)$ \\
\hline \multicolumn{2}{|c|}{ Smoke tobacco } \\
\hline No & $108(51.9)$ \\
\hline Yes & $25(12.0)$ \\
\hline Unknown & $75(36.1)$ \\
\hline \multicolumn{2}{|c|}{ Consume alcohol } \\
\hline No & $89(42.8)$ \\
\hline Yes & $44(21.2)$ \\
\hline Unknown & $75(36.1)$ \\
\hline \multicolumn{2}{|c|}{ Past medical history } \\
\hline No & $72(34.6)$ \\
\hline Yes & $61(29.3)$ \\
\hline Unknown & $75(36.1)$ \\
\hline \multicolumn{2}{|c|}{ Daily medication use } \\
\hline No & $98(47.1)$ \\
\hline Yes & $35(16.8)$ \\
\hline Unknown & $75(36.1)$ \\
\hline \multicolumn{2}{|c|}{ Body temperature (degrees Celsius) } \\
\hline Under 37 & $154(74.0)$ \\
\hline $37.0-37.5$ & $48(23.1)$ \\
\hline Over 37.5 & $4(1.9)$ \\
\hline Unknown & $2(1.0)$ \\
\hline \multicolumn{2}{|l|}{ Hypertension } \\
\hline No & $126(60.6)$ \\
\hline Yes & $80(38.5)$ \\
\hline Unknown & $2(1.0)$ \\
\hline \multicolumn{2}{|c|}{ Body Mass Index } \\
\hline Under 18.4 & $33(15.9)$ \\
\hline $18.5-24.9$ & $122(58.7)$ \\
\hline $25-29.9$ & $37(17.8)$ \\
\hline Over 30 & $7(3.4)$ \\
\hline Unknown & $9(4.3)$ \\
\hline
\end{tabular}

Hypertension: systolic blood pressure above 140 or diastolic blood pressure above 90 .

Fisher's exact test). The rate of high BMI was similar in both genders ( $P=0.609$; Fisher's exact test). Eighty hypertensive patients were identified; however, only 8 patients were identified as taking medication for hypertension.
Table 3 shows the five most frequent medical complaints identified by the participants and the five most frequent diagnoses made by the doctors, with the exception of hypertension. The most common complaint mentioned by respondents was headache (18.3\%), followed by lower back pain (14.4\%). The most frequent diagnosis provided by the doctors was arthritis (16.8\%), followed by gastroenteritis $(13.5 \%)$.

\section{Discussion}

Health care needs assessments regarding NCDs in poor rural areas in Cambodia are vital. This study found that the major medical complaint was chronic pain and medical needs for consultation for chronic pain are high in such poor rural areas. Poor people living in rural areas have unmet needs for medical care for chronic diseases ${ }^{8}$. It is necessary to develop a system in which health care for treating chronic diseases is accessible to all.

The proportion of people who have lifestyle-related disease risk factors such as smoking tobacco and alcohol consumption might have regional differences. In comparison to a previous (2008) nation-wide survey on smoking in Cambodia, smoking rates among participants in our study were low $37.9 \%$ among men and $2.8 \%$ among women in this survey, $48.0 \%$ among men and $3.6 \%$ among women in the 2008 survey ${ }^{9}$. However, the proportion of people who consume alcohol was high in our study compared to a previous (2013) survey on alcohol consumption in Cambodia $(51.7 \%$ among men and $18.1 \%$ among women in this survey, $35.4 \%$ among men and $7.5 \%$ among women in the 2013 survey $)^{10}$. When considering these results, it is necessary to consider not only prevalence rates in the whole country but also rates in each region in order to provide effective health education for residents. In Cambodia, alcohol consumption and tobacco smoking rates were overwhelmingly high among men $^{9}{ }^{10}$. In addition, household air pollution is one of the biggest risk factors in Cambodia ${ }^{4}$. In fact, most residents in the rural area of Prey Veng province use wood as fuel ${ }^{5}$. These risk factors might be the cause of difficulty in breathing, which was listed among the major complaints by residents in this survey. It is vital to reduce these risk factors by educating patients to improve their lifestyle habits.

One limitation of this study was that we did not obtain information regarding the type of tobacco habit: cigarette smoking, pipe smoking, or chewing tobacco. Chewing tobacco is widely used among Cambodian women"); however, we did not ask about chewing tobacco in this study. Another limitation is that the sampling in this study was not random, and a selection bias exists because almost all participants had a medical complaint that required consultation with a doctor. Besides, the sample size was small, and the numbers of missing cases was high for smoking, alcohol consumption, medication use, 
Table 2 Participant characteristics for each age and gender among participants living in Kaoh Peam Reang area, Prey Veng province, Cambodia in $2019(n=208)$

\begin{tabular}{|c|c|c|c|c|c|c|c|}
\hline & \multirow{2}{*}{$\begin{array}{c}\text { Alcohol } \\
n(\%)\end{array}$} & \multirow{2}{*}{$\begin{array}{c}\text { Smoker } \\
n(\%)\end{array}$} & \multirow{2}{*}{$\begin{array}{c}\text { Hypertension } \\
n(\%)\end{array}$} & \multicolumn{4}{|c|}{ Body Mass Index n (\%) } \\
\hline & & & & Under 18.4 & $18.4-24.9$ & $25-29.9$ & Over 30 \\
\hline \multicolumn{8}{|l|}{ Age } \\
\hline Under 19 & $0(0)$ & $0(0)$ & $1(7.1)$ & $6(50.0)$ & $6(50.0)$ & $0(0.0)$ & $0(0.0)$ \\
\hline $20-34$ & $3(21.4)$ & $1(7.1)$ & $7(29.2)$ & $2(8.3)$ & $14(58.3)$ & $6(25.0)$ & $2(8.3)$ \\
\hline $35-49$ & $22(66.7)$ & $8(24.2)$ & $16(35.6)$ & $4(8.9)$ & $27(60)$ & $12(26.7)$ & $2(4.4)$ \\
\hline $50-64$ & $12(27.9)$ & $7(16.3)$ & $30(46.9)$ & $5(8.1)$ & $38(61.3)$ & $16(25.8)$ & $3(4.8)$ \\
\hline $65-80$ & 7 (21.2) & $6(18.2)$ & $25(51.0)$ & $13(27.7)$ & $31(66.0)$ & $3(6.4)$ & $0(0.0)$ \\
\hline Over 80 & $0(0)$ & $2(50)$ & $1(25.0)$ & $1(25.0)$ & $3(75.0)$ & $0(0.0)$ & $0(0.0)$ \\
\hline \multicolumn{8}{|l|}{ Gender } \\
\hline Male & $30(51.7)$ & $22(37.9)$ & $50(59.5)$ & $14(17.1)$ & $53(64.6)$ & $12(14.6)$ & $3(3.7)$ \\
\hline Female & $13(18.1)$ & $2(2.8)$ & $29(25.2)$ & $17(15.2)$ & $66(58.9)$ & $25(22.3)$ & $4(3.6)$ \\
\hline
\end{tabular}

$(\%)=$ The number of participant who answered (or measured) yes / total number of participants in each age and gender group.

Hypertension: systolic blood pressure above 140 or diastolic blood pressure above 90 .

Table 3 Five major medical complaints and five major diagnoses among participants living in Kaoh Peam Reang area, Prey Veng province, Cambodia in $2019(n=208)$

\begin{tabular}{lc}
\hline & $\mathrm{n}(\%)$ \\
\hline Medical complaints of participants & \\
Headache & $38(18.3)$ \\
Lower back pain & $30(14.4)$ \\
Foot/hand pain & $29(13.9)$ \\
Joint pain & $21(10.1)$ \\
Breathing difficulties & $21(10.1)$ \\
Medical diagnoses (except hypertension) & \\
Arthritis & $35(16.8)$ \\
Gastroenteritis & $28(13.5)$ \\
Backache & $23(11.1)$ \\
Tension type headache & $14(6.7)$ \\
Urinary tract infection & $11(5.3)$ \\
\hline
\end{tabular}

and past medical history variables. Furthermore, we did not survey household air pollution although it is a major risk factor in Cambodia ${ }^{4}$. Despite these limitations, this study was valuable as a pilot project to identify the health care needs relating to NCDs among poor rural island residents.

\section{Conclusion}

The medical needs for doctor consultations regarding chronic disease and chronic pain might be higher in poor rural areas. It is vital to work towards developing a system in which health care for treating chronic diseases is accessible to all, and to tailor-make health education for each district's residents. Further research and additional countermeasures that can improve the health problems of rural residents are needed.

Funding sources: This research did not receive any specific grant from any funding agency in the public, commercial, or not-for-profit sector.

Disclosures: All the authors report no proprietary or commercial interest in any product mentioned or concept discussed in this article.

Author contributions: All the authors made a substantial contribution to this research. YK, MT, and HC contributed to writing the paper. All members contributed to the study design, data collection, and coordination with local stakeholders.

\section{Acknowledgment}

The authors are thankful to the residents and staff in Kaoh Peam Reang who cooperate in the free health checkups. In addition, the authors thank all the people who cooperated in the free health check-ups and this study. The authors thank Sunrise Japan Hospital staff who engage in social responsibility activities. The authors would like to say thank you to Professor Andy Crump who gave us constructive advice for improving our English and our writing. 


\section{References}

1. World Health Organization World Health Statistics 2018. 2018; https://apps.who.int/iris/bitstream/handle/10665/272596/9789241565585-eng.pdf?ua=1. Accessed April 16, 2020.

2. World Health Organization Global Action Plan for the Prevention and Control of Noncommunicable Diseases 2013-2020. 2013; https://apps.who.int/iris/ bitstream/handle/10665/94384/9789241506236_eng.pdf?sequence=1. Accessed April 20, 2020.

3. World Health Organization Noncommunicable Diseases. 2018; https://www.who.int/news-room/fact-sheets/detail/noncommunicable-diseases. Accessed April 16, 2020.

4. GBD 2015 Risk Factors Collaborators Global, regional, and national comparative risk assessment of 79 behavioural, environmental and occupational, and metabolic risks or clusters of risks, 1990-2015: a systematic analysis for the Global Burden of Disease Study 2015 [published correction appears in Lancet 2017 Jan 7, 389(10064).e1]. Lancet 2016; 388: 1659-1724. [Medline] [CrossRef]

5. National Institute of Statistics, Directorate General for Health, and ICF International Cambodia Demographic and Health Survey 2014. 2015; https:// dhsprogram.com/pubs/pdf/fr312/fr312.pdf. Accessed April 16, 2020.

6. Bureau of Health Economics and Financing, Department of Planning and Health Information Annual Health Financing Report 2015. 2015; https://www. dfat.gov.au/sites/default/files/cambodia-ministry-of-health-annual-health-financing-report-2014.PDF. Accessed April 16, 2020.

7. Ministry of Health National Multisectoral Action Plan for The Prevention and Control of Non-communicable Disease 2018- 2027. 2018; http://moh.gov. kh/content/uploads/2017/05/NMAP-NCD_-13-06-2018-Signed_En.pdf. Accessed April 20, 2020.

8. Ministry of Health National Strategic Plan for the Prevention and Control of Noncommunicable Diseases. 2013; https://www.iccp-portal.org/system/files/ plans/KHM_B3_NSP-NCD\%202013-2020_Final\%20approved.pdf, Accessed April 20, 2020.

9. Singh PN, Yel D, Sin S, et al. Tobacco use among adults in Cambodia: evidence for a tobacco epidemic among women. Bull World Health Organ 2009; 87: 905-912. [Medline] [CrossRef]

10. Banta JE, Addison A, Job JS, et al. Patterns of alcohol and tobacco use in Cambodia. Asia Pac J Public Health 2013; 25(Suppl): 33S-44S. [Medline] [CrossRef] 